\title{
Contour Tracking in Echocardiographic Sequences without Learning Stage: Application to the 3D Reconstruction of the Beating Left Ventricule
}

\author{
Marie-Odile Berger ${ }^{1}$, Goetz Winterfeldt ${ }^{1}$, and Jean-Paul Lethor ${ }^{2}$ \\ 1 Loria/Inria-Lorraine, BP 101 \\ F-54600 Villers-les-Nancy, France \\ berger@loria.fr \\ 2 CHU Brabois, Nancy, France
}

\begin{abstract}
In this paper we present a contour tracker on echographic image sequences. To do this, we use a hierarchical approach: we first compute a global estimation of the ventricular motion. Then we use a fine tuning algorithm to adjust the detection of the ventricular wall. The global estimation is based on a parametric motion model with a small number of parameters. This allows us to compute the motion in a robust way from the velocity computed at each point of the contour.

Results are presented demonstrating tracking on various echographic sequences. We conclude by discussing some of our current research efforts.
\end{abstract}

\section{Introduction}

Parameters of cardiac functions can be drawn from the analysis of echocardiographic image sequences, especially the motion of the ventricular wall, heart wall thickness and shape parameters. These analyses are based on the detection of the left ventricular internal wall edge. Usually, the ventricular edges are manually traced by the physician but this operation is time consuming if an entire cardiac cycle must be analyzed. Hence, there is considerable interest in developing methods to perform automatic extraction of the ventricular contours in a sequence.

Several authors have recently proposed contour trackers which rely on a preliminary learning stage [3]. This allows them to restrict the class of allowable motion (shape deformations) to an admissible set that has been learned on a training data set of the patient to be examined. As the variability of the ventricular deformation between patients can be large, the allowable deformations for a given patient is likely to be not suited for another one.

That is the reason why we attempt to measure cardiac motion directly on grey level images. Within the computer vision community, considerable attention has been given to tracking and understanding object motion. Methods for computing motion can roughly be categorized as either differential or matching methods. However, the performance of these techniques are not the same: 
the accuracy, reliability and density of the computed velocity field can differ significantly according to the technique used.

- differential techniques: the common assumption is that the observed intensity is constant over time. The constant brightness assumption can be formally stated as $I(x, y, t)=I(x+\delta x, y+\delta y, t+\delta t)$. Expanding the image brightness function in a Taylor'e series around the points $(x, y)$ we obtain the gradient constraint equation:

$$
\nabla I(x, y, t) \cdot v+I_{t}(x, y, t)=0
$$

It is a single equation which allows us to compute the normal optical flow $v^{\perp}$. Due to the finite differences used to approximate derivatives in the consistancy equation (1), differential techniques are thus inacurrate for large displacements. This may cause problems to track the mitral valve because this structure is thin and can undergo large motions.

- matching techniques [5]: such approaches define velocity $v$ as the shift that yields the best fit between image regions at different times. Finding the best match amounts to minimize a similarity measure over $v$ such as the sum of squared differences:

$$
v=\operatorname{argmin}_{\left\{v=\left(v_{x}, v_{y}\right)\right\}} \sum_{i=-w}^{i=w} \sum_{j=-w}^{j=w}\left[I(x+i, y+j, t)-I\left(x+i+v_{x}, y+j+v_{y}, t+1\right)\right]^{2}
$$

However, as there are no specific points on the ventricular wall, the peak of the correlation is not always clearly discernible. It is therefore difficult to estimate point wise motion. Consider for instance Fig.1: The left image shows the ventricular wall traced by an expert. The right image shows the correspondences given by the correlation (original points are drawn in black and the detected points are drawn in white). In regions where gradient information is missing (right part of the ventricle), results are of course very poor. On the left side, the computed motion is more reliable. In contrast to differential techniques, large displacements can be handled with correlation based methods provided that the size $w$ of the window correlation is large enough. However, the reliability of the computed motion is not very good.

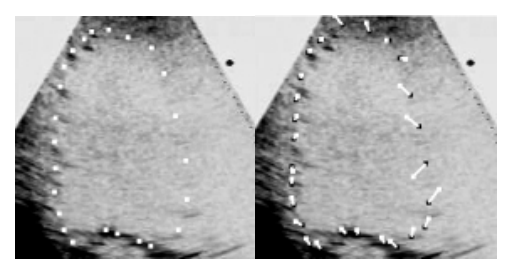

Fig. 1. Correspondences given by correlation: (a) the ventricular wall in a given frame (b) the motion computed with correlation in the next frame. 
To conclude, due to the weak signal to noise ratio in echocardiographic images, the computed velocity is not relevant at several places of the contour. These measurements can not therefore be used as is for the ventricular wall detection. Following previous works on deformable structures [2] we therefore use a hierarchical algorithm; we first compute a global estimation of the ventricular deformation. Then we use a fine tuning deformation to adjust the details. The global estimation is based on a parametric motion model with a small number of parameters $(4,5$ or 6$)$. These parameters are estimated in a robust way from the velocity field computed at each point of the contour. From this estimation, active contours models are used to detect the ventricular wall.

The motion models we use are described in section 2. The overview of our tracker is given in section 3. Section 4 describes our method for computing motion. Finally, we show in section 5 experimental results that demonstrate the validity of our approach.

\section{Which Motion Model?}

In order to describe the ventricular wall motion properly, we tested three motion models: similarity, affinity and affine model. The similarity model

$$
\begin{aligned}
& X=s \cos \theta x-s \sin \theta y+t_{x} \\
& Y=s \sin \theta x+s \cos \theta y+t_{y}
\end{aligned}
$$

describes a contraction $(s<1)$ or an expansion $(s>1)$ of the ventricle with respect to a central point.

The affinity model:

$$
\begin{aligned}
& X=s_{1} \cos \theta x-s_{2} \sin \theta y+t_{x} \\
& Y=s_{1} \sin \theta x+s_{2} \cos \theta y+t_{y}
\end{aligned}
$$

uses two different scale factors $s_{1}$ and $s_{2}$ in two orthogonal directions. At first sight, this is well suited for describing the ventricle motion: indeed, the main motion of the ventricle is perpendicular to the long axis of the ventricle. In addition, there is a small motion along the long axis.

The affine model:

$$
\begin{aligned}
& X=a x+b y+t_{x} \\
& Y=c x+d y+t_{y}
\end{aligned}
$$

is more general and allows various motions to be handled.

In the following, the motion model is denoted $f_{p}$ where $p$ is the set of parameters models. $p=\left[s, \theta, t_{x}, t_{y}\right]$ for a similarity, $p=\left[s_{1}, s_{2}, \theta, t_{x}, t_{y}\right]$ for an affinity and $p=\left[a, b, c, d, t_{x}, t_{y}\right]$ for an affine model. The advantages and drawbacks of these three models are discussed in the section devoted to the results.

\section{Overview of Tracker}

The tracker is initialized with the contour traced by the expert in the first frame of the sequence. Once initialized, the tracker operates in a loop following three major steps: prediction, motion computation and adjustment (Fig. 2). 


\section{Computation of an initial guess using correlation based methods. \\ 2. Iterative computation of the velocity field using motion model. \\ 3. Adjustment of the ventricular wall with snakes.}

Fig. 2. Block diagram of the tracker

The first step aims at computing an initial guess in order to overcome problems stemming from small structures undergoing large motion as the mitral valve. Let $\left\{M_{i}\right\}_{1<i<N}$ be the points of the contour detected in a given frame. Using cross correlation, these points can be matched with points $P_{i}$ in the next frame. If $f_{p}$ is the motion model used, a first estimation of the ventricle in the next frame is obtained by minimizing the distance between the two curves $C=\left\{f_{p}\left(M_{1}\right), \ldots f_{p}\left(M_{N}\right)\right\}$ and $C^{\prime}=\left\{P_{1}, \ldots, P_{N}\right\}$. As the correspondence given by the correlation process is not always relevant, we use the closest point distance and we compute the optimal parameters $p$ minimizing

$$
\min _{p} \sum \operatorname{distance}\left(f_{p}\left(M_{i}\right), C^{\prime}\right)
$$

where $\operatorname{distance}\left(f_{p}(M), C^{\prime}\right)=\min _{i}\left\|f_{p}(M), P_{i}\right\|$. Hence, $\left\{f_{p}\left(M_{1}\right), \ldots f_{p}\left(M_{n}\right)\right\}$ is a first rough estimation of the ventricular wall.

From this initial guess, the ventricle motion is computed iteratively using only normal optical flow. This computation is described in section 4 .

Finally, snakes [4] are used from the predicted position of the ventricle. In most cases, this position is sufficiently close to the ventricular boundary so that the snake converges successfully.

The main steps of our algorithm are illustrated in figure 3. Fig. 3.a shows the ventricle border detected in a given frame. Fig. 3.b exhibits the motion (Step 1+2) computed between the two frames using the affinity model. Fig. 3.c shows the results of the adjustment after the snake process.

\section{Motion Computation}

Let $C$ be the ventricular contour detected in a given frame $I_{1}$. Our aim is to compute the motion of $C$ between two consecutive frames $I_{1}$ and $I_{2}$.

We implemented a weighted least squares fit of local first-order constraint (1) to a global model of $v$ on the ventricular contour by computing $\hat{p}_{0}$ which minimizes

$$
\sum_{1 \leq i<N}\left|\left(\overrightarrow{M_{i} f_{p}\left(M_{i}\right)} \cdot n_{i}\right) n_{i}-v^{\perp}\left(M_{i}\right)\right|^{2}
$$

where $n_{i}$ is the unit normal to $\mathcal{C}$ at point $M_{i}$ and $v^{\perp}$ is the normal flow computed from (1). This way, we calculate the motion $\hat{p}_{0}$ which yields the best fit with 

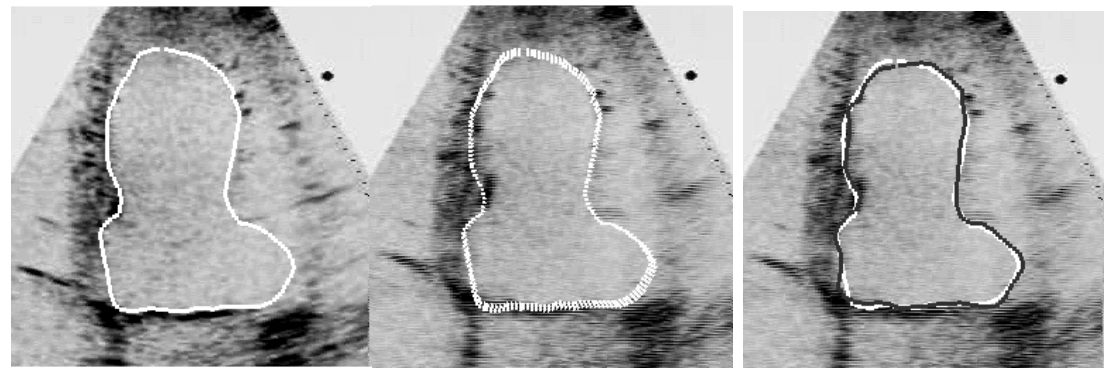

Fig. 3. The main steps of the tracker: (a) the contour detected in a given frame (b) the computed motion (c) prediction (in white) and result of the detection (in black).

the motion measurements computed with differential techniques. The use of a global motion of the curve is very important because it permits us to override divergence trends at erroneous flow points.

As the optical flow does not match the true displacement, we refine the estimation iteratively in the following way: we compute the normal optical flow $v_{1}^{\perp}$ on the curve $f_{\hat{p}_{0}}\left(M_{i}\right)_{\{1 \leq i \leq N\}}$ between the registered image $I_{1}\left(f_{\hat{p}_{0}}^{-1}\right)$ and $I_{2}$. Resolving (2) with $v=v_{1}$ then allows us to compute $f_{\hat{p}_{1}}$. Hence, $f_{\hat{p}_{1}} \circ f_{\hat{p}_{0}}$ is a better estimate of the motion and so on... Successive infinitesimal refinements $f_{\hat{p}_{q}} \circ \ldots \circ f_{\hat{p}_{0}}$ gives an accurate estimation of the motion field. The prediction obtained is generally close to the true ventricular wall and the snake process can be applied successfully.

\section{Results}

Our method has been tested within a project aiming at 3D reconstruction of the beating left ventricle [6]. The system consists of a probe rotating around its axis. For each rotation of the probe, an entire heart contraction is recorded at a 25 frames/sec rate. We then acquire a matrix of images. Its size is $8 \times 9$ because we only acquire images between end diastolic and end systolic ( 8 images) and the probe rotation is 20 degrees.

First, we compare the performance of the tracking algorithm using the three motion models we use: similarity, affinity and affine model. A frame by frame visual comparison of tracking using these three models showed that the affinity model generally gives superior results in term of how closely the tracker followed the ventricle. Indeed, it appears that the similarity model does not manage to encompass the variability of the shape deformation. On the other hand, the affine model is not sufficiently restrictive when the noise level is high and the prediction step may be erroneous. Hence, the use of affinity appears as a good compromise.

We tried to quantify the degree of improvement by comparing our results to the ventricle manually traced by an expert in some sequences. Note that these 
traces can only be considered as indicative and not as ground truth. Indeed, there is a large variability between the traces outlined by different experts [1].

\section{Error Metric}

We compare the areas enclosed by the expert detection and the computed detection. We think that this measure is more appropriate because the area of the ventricle is an important clinical parameter to evaluate the left ventricular function. More precisely, if $\mathcal{A}_{\text {comp }}$ (resp $\left.\mathcal{A}_{\text {expert }}\right)$ is the area enclosed by the computed (resp. expert) detection, the metric used is defined as

$$
\text { area error }=\frac{\#\left\{x \in \mathcal{A}_{\text {comp }} \text { and } x \notin \mathcal{A}_{\text {expert }}\right\} \cup\left\{x \notin \mathcal{A}_{\text {comp }} \text { and } x \in \mathcal{A}_{\text {expert }}\right\}}{\# \mathcal{A}_{\text {expert }}}
$$

where \#E denotes the cardinal number of $E$.

\section{Tracking experiments}

Four matrix database have been outlined by the expert. This amounts to outline the ventricle in $8 \times 9$ images for each patient. In order to test our algorithm, we considered the contour $c_{i \times j}$ traced by the expert in frame $i$ of the sequence which corresponds to the probe angle $j \times 20$ degrees. We computed the distance between the results given by the algorithm in the next frame to the contour $c_{i+1 \times j}$ traced by the expert. The results are averaged on all the images which have been outlined by the expert in the database.

This computation was performed for the three motion models and the results are shown in table 5 . The area error in percentage is around $6 \%$.

These results prove that the affinity model is slightly more appropriate than the two others. Not surprisingly, the affine model gives the worst results because of its excessive flexibility. The standard deviation is the highest for this model; when we look at the results (Fig.4, 6), we can see that results are sometimes very good with this models. Unfortunately they are sometimes poor for high noise level.

We now give tracking results for different patients. Fig 4 shows snapshot views of tracking using the affinity models. The detected curve is shown in white whereas the expert trace is drawn with black points. The visual impression is good. Fig 5.a plots the evolution of the area errors with the three motions models. In this case, the area errors are rather similar and the results obtained in the last frame are close too (Fig. 5.b).

Fig. 6 shows tracking results for another patient. Tracking results are good except at the end of the sequence because the process is unable to handle the ventricular deformation in the right direction.

\begin{tabular}{||l|c|c|c|c||}
\hline Motion model & $\begin{array}{c}\text { area errors (in \%) } \\
\text { Mean }\end{array}$ & $\begin{array}{c}\text { area errors } \\
\text { standard deviation }\end{array}$ & min error & max error \\
\hline Similarity & 6.0837 & 1.6884 & 3.6258 & 10.5606 \\
\hline Affinity & 5.8903 & 1.5169 & 3.8016 & 10.0563 \\
\hline Affine & 6.2169 & 1.9765 & 3.6323 & 14.1187 \\
\hline
\end{tabular}

Table 1. Comparison of the tracking error for the three motion models. 


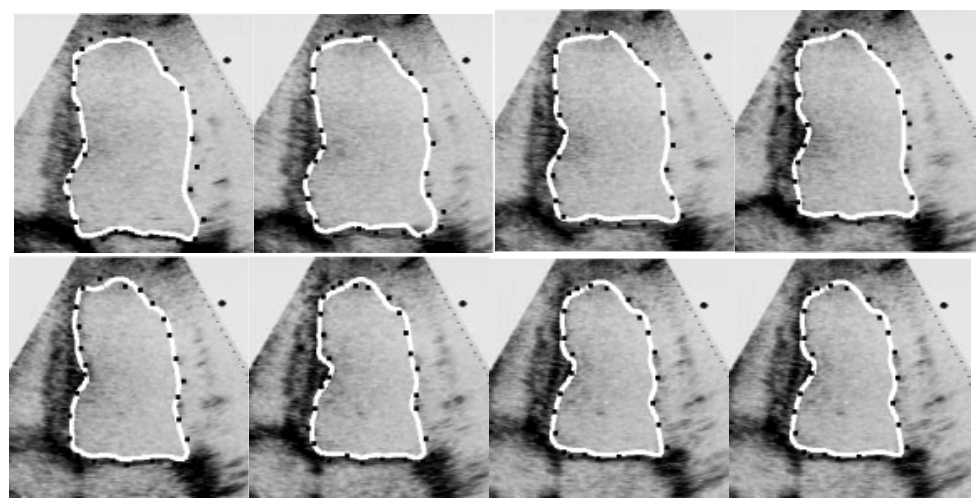

Fig. 4. Tracking results: the tracked ventricle is shown in white and the trace of the expert is shown with black points.
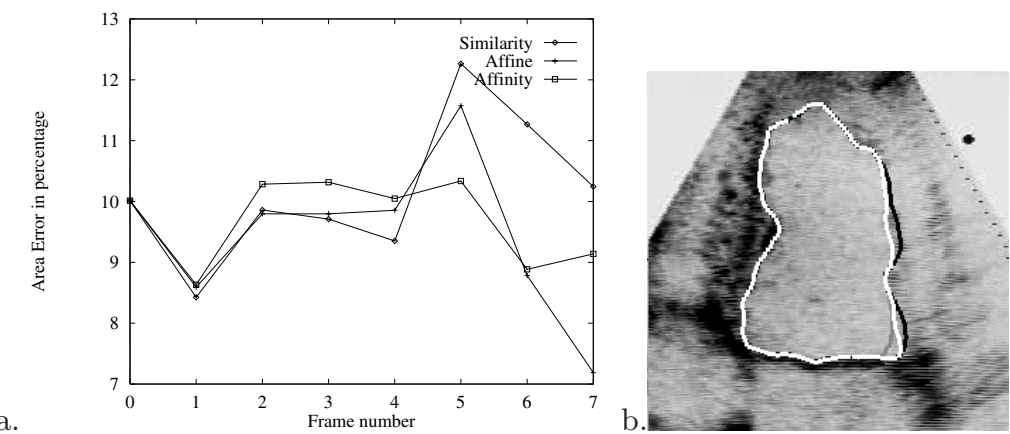

Fig. 5. (a) Plot of the area errors trough the sequence for the three motion models (b) results of the tracking in the last frame: similarity is in gray, affinity is in white and affine in black.

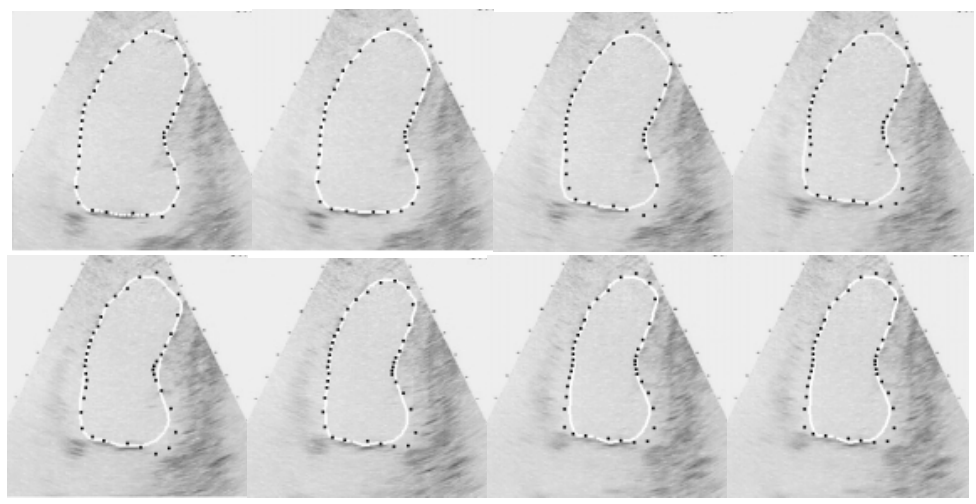

Fig. 6. Tracking results: the tracked ventricle is shown in white and the trace of the experts is shown with black points. 
$3 \mathrm{D}$ reconstruction Fig. 7 exhibits the $3 \mathrm{D}$ construction of the left ventricle with the patient used in Fig. 6 .
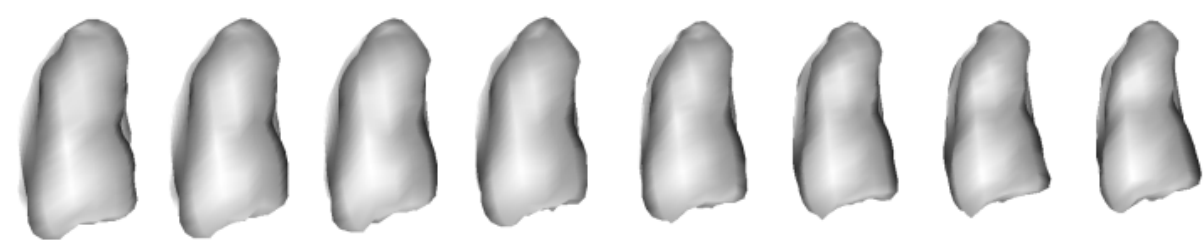

Fig. 7. 3D reconstruction of the beating left ventricle between end diastolic and end systolic.

\section{Discussion and Conclusion}

To conclude we have presented a new approach to tracking on echocardiographic images. Our approach, which combines both motion based approach and fine adjustment with snakes, give promising results. The use of this hierarchical approach allows us to compute a reliable estimation of the velocity which can be used successfully as initial guess for the adjustment stage.

It is clear that further investigation should be made into the problem of ventricular adjustment. In fact, the snakes can be attracted by structures which do not belong to the endocardium. Indeed, gradient information obtained from echographic images is always incomplete because of drop outs. This problem especially appears because of shadowing effect caused by the ribs. We currently investigate how the spatial coherency of our data can be used to overcome the above problems. Indeed, some structures of the endocardium which are missing in a scan plane are likely to be present in the neighbored scan planes. That is the reason why we want to achieve the adjustments stage in the $3 \mathrm{D}$ space instead of the $2 \mathrm{D}$ space.

\section{References}

1. V. Chalna and Y. Kim. A methodology for evaluation of boundary detection algorithms on medical images. IEEE Transactions on Medical Imaging, 16(5):642652, December 1997. 513

2. M. Chen, T. Kanade, H. Rowley, and D.Pommerleau. Anomaly Detection through Registration. In CVPR' 1998, 1998. 510

3. G. Jacob, A. Noble, and A. Blake. Robust Contour Tracking in Echographic Sequences. In ICCV 98, Bombay (India), pages 408-413, January 1998. 508

4. M. Kass, A. Witkin, and D. Terzopoulos. Snakes: Active Contour Models. International Journal of Computer Vision, 1:321-331, 1988. 511 
5. C. Lamberti, P. Botazzi, and A. Sarti. Region Based Matching Field Computation in 2D Echocardiography. In Computers in Cardiology, 1993, pages 739-742, 1993. 509

6. J.-P. Lethor, G. Winterfeldt, M.-O. Berger, M. Handschuhmacher, and I. Marcon. A Fast automated System for Four Dimensional Reconstruction of the left Ventricle Using Transthoracic Echographic Images. In Computers in Cardiology, 1997. 512 